\title{
The Effectiveness of the Polarized Low-Level Laser in the Treatment of Patients With Myofascial Trigger Points in the Trapezius Muscles
}

\author{
Dadollah Shahimoridi ${ }^{1}$, Seyed Ali Shafiei ${ }^{2 *}$, Bahram Yousefian ${ }^{3}$ \\ 'Department of Basic Sciences, School of Medicine, Rafsanjan University of Medical Sciences, Rafsanjan, Iran \\ ${ }^{2}$ Neuroscience Research Center, School of Medicine, Qom University of Medical Sciences, Qom, Iran \\ ${ }^{3}$ Department of Radiology, School of Allied Medicine, Rafsanjan University of Medical Sciences, Rafsanjan, Iran
}

\section{*Correspondence to Seyed Ali Shafiei, Neuroscience Research Center, School of Medicine, Qom University of Medical Sciences, Qom, Iran Tel: +982533209071 Fax: +982533209127; Email: sashafiei@muq.ac.ir}

Published online January 18,2020

\begin{abstract}
Introduction: This study assessed the effect of polarized low-level laser therapy (PLLLT) on the treatment of myofascial trigger points (MTrPs) in the trapezius muscles. Its effectiveness in pain reduction was compared to low-level laser therapy (LLLT).

Methods: Sixty-four patients with MTrPs were randomly divided into 2 groups, namely PLLLT and LLLT. Each patient received treatment for a period of 2 weeks, 5 sessions a week. The intensity of laser irradiation to the skin surface was $6 \mathrm{~J} / \mathrm{cm}^{2}$. The system exit power was $160 \mathrm{mw}$ at a $755 \mathrm{~nm}$ wavelength. The visual analog scale (VAS) for the 1st, 5th and 10th sessions was analyzed through two-way repeated measures ANOVA.

Results: Increasing the number of treatment sessions was effective in reducing pain intensity $(P>0.05)$. The effect of LLLT on pain reduction was significantly greater than that of PLLLT $(P>0.05)$. Referred pain (RP), the limitation of neck movement (LNM), the presence of muscular taut band (PMTB) and the sensitivity of muscular taut band (SMTB) were reduced significantly by the end of the 10th session, compared with participants' condition at the beginning of the 1st session in both groups.

Conclusion: PLLLT and LLLT can effectively treat MTrPs in the trapezius muscles and they reduce RP, LNM, PMTB, and SMTB in particular. However, the effect of the LLLT was significantly greater than that of PLLLT. In accordance with the observed results, LLLT is recommended as an effective method for treating MTrPs.

Keywords: Low-level laser therapy; Myofascial pain; Polarized light.
\end{abstract}

\section{Introduction}

Epidemiologic studies of musculoskeletal pain syndromes show that myofascial trigger points (MTrPs) pain is very common. More than $55 \%$ of neck pain and $85 \%$ of lumbar and back pain are related to $\mathrm{MTrPs}^{1,2}$ The trigger point is a hyperirritable spot located within the taut band of the skeletal muscle fascia, described as a small palpable hypersensitive area that may be causing referred pain (RP) upon palpation and the limitation of motion., ${ }^{2,3}$ MTrPs and related pain syndromes usually lead to poor physical performance, poor sleep quality, and low quality of life. ${ }^{4,5}$ The treatment modalities of trigger points are multiple and include pharmacotherapy (antidepressant, anticonvulsants, muscle relaxants, local anesthetics, etc) and non-pharmacological treatments (massage therapy, dry needling, stretching exercises, ultrasound, etc).${ }^{6-8}$ One of the debated treatment modalities, despite its common use, is low-level laser therapy (LLLT) ${ }^{9-14}$ that is used in the treatment of many diseases. ${ }^{15-20}$ Imani et al and Munguia et al concluded that almost $60 \%$ of the literature between 2000 to 2018 demonstrated that LLLT was useful in reducing pain and trigger point sensitivity, improving the quality of life. ${ }^{21,22}$ Momenzadeh et $\mathrm{al}^{23}$ reached the same result. $^{24}$

Several mechanisms of action have been proposed for pain reduction by LLLT. LLLT may lead to the reduction of skin resistance, enhancing circulation in small blood vessels at the MTrPs, improving oxygenation of the hypoxic cells at the trigger points, and facilitating the removal of waste material from the MTrPs area. The normalization of small vessel blood circulation will allay blood deficiency at the pain source and restrain its spread in the region. ${ }^{24-26}$ Some authors have suggested that LLLT exerts its therapeutic effects through other mechanisms such as collagen reproduction, anti-inflammatory effect, the stimulation of the peripheral nerves and tranquilizer

Please cite this article as follows: Shahimoridi D, Shafiei SA, Yousefian B. The effectiveness of the polarized low-level laser in the treatment of patients with myofascial trigger points in the trapezius muscles. J Lasers Med Sci. 2020;11(1):14-19. doi:10.15171/jlms.2020.04. 
effect. $^{27,28}$

There is another noninvasive and non-pharmaceutical method for pain treatment based on polarized conventional light (from violet [about $400 \mathrm{~nm}$ ] to nearinfrared [about $2000 \mathrm{~nm}$ ]). ${ }^{29-31}$ However, non-polarized light lacks this therapeutic effect, likely due to electrical field alignment in irradiation with polarized light (PL). $\mathrm{PL}$ is widely used in the treatment of various types of wounds, including burn wounds to the skin and pressure ulcer. $^{32,33}$

Considering these 2 mentioned modalities (PL and LLLT) in the treatment of pain syndromes, the goal of this study was to examine the effectiveness of polarized lowlevel laser therapy (PLLLT) in the reduction of pain of MTrPs in the trapezius. We also compared its effectiveness in pain control with LLLT. Given the controversy in the literature over the efficacy of LLLT in the treatment of MTrPs, the current study was initiated to examine the treatment of MTrPs.

\section{Materials and Methods}

\section{Subjects}

Sixty-four patients with MTrPs were selected from patients who were referred to Ali-ibn Abi Talib Hospital in Rafsanjan. According to the criteria established by Simunovic, ${ }^{26}$ physical findings in 5 major criteria and 1 of 3 minor criteria were present for the diagnosis of MTrPs. The major criteria are local pain, RP originating from trigger points, the presence of the palpable and taut muscular band in involved muscles, and reduction in the range of motion and sensitivity along the length of the taut band. The minor criteria are the creation of fast muscular contraction response, the spontaneous alteration of pain sensation when pressing trigger points, and muscular pain associated with stretching. ${ }^{26}$ To achieve sample size equivalency in the groups, through the replacing-block method, the patients were randomly divided into two groups, PLLLT and LLLT (Figure 1).

Physical examination revealed a palpable taut muscular band of the trapezius and muscle. Laser irradiation was applied to all painful trigger points in each patient. The patients with these symptoms and a visual analog scale (VAS) grade greater than $50 \mathrm{~mm}$ were selected to participate in this research (Table 1). Informed consent was obtained from each participant. All patients' information was kept confidential. The patients had autonomy to participate or to leave the study at any time.

\section{Polarized Laser}

The exit light of the laser device was polarized by a $5 \times 6$ $\mathrm{cm}^{2}$ polaroid sheet film (PSF).

The PSF was a thin, flexible and transparent sheet that was sandwiched between two pieces of transparent Plexiglas with $2 \mathrm{~mm}$ thickness. The PSF was placed in front of the laser output for making polarized laser beam. A Genova spectrophotometer (Jenway, Bibby Scientific,

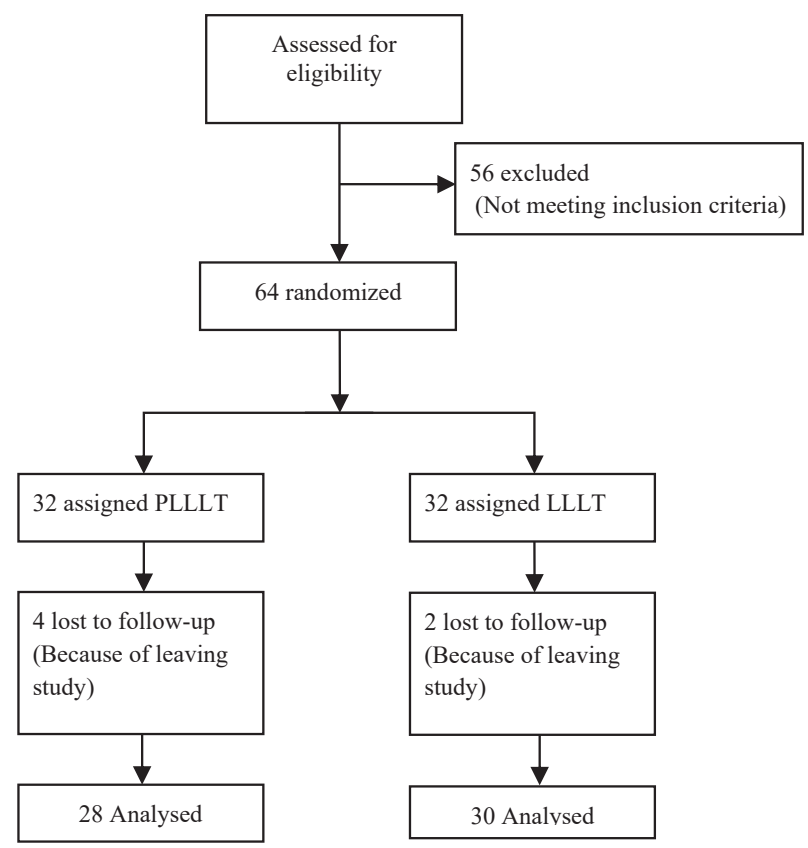

Figure 1. Flow Diagram of the Participants.

Table 1. Demographic Characteristics of the Patients (First Session)

\begin{tabular}{lcc}
\hline & LLLT & PLLLT \\
\hline Age $(\mathrm{y})$ & $43.87 \pm 11.41$ & $44.62 \pm 12.32$ \\
Pain duration (months) & $6.68 \pm 3.53$ & $7.34 \pm 3.58$ \\
Pain intensity $\left(\mathrm{mm}^{*}\right)$ & $77.50 \pm 10.02$ & $77.66 \pm 8.56$ \\
Gender & & \\
Male & $9(32.1 \%)$ & $12(40 \%)$ \\
Female & $19(67.9 \%)$ & $18(60 \%)$ \\
\hline
\end{tabular}

LLLT: low-level therapy laser; PLLLT: polarized low-level therapy laser.

*Visual Analog Scale (VAS) score in millimeters.

Stafford shire, UK) was used to measure the reduced light transmission through the Plexiglas sheets. In the absence of PSF, a pair of Plexiglas sheets was placed in front of the exiting low-level laser (LLL) to even out the rate of weakening because the present Plexiglas sheets had similarly been used with the polarized low-level laser (PLLL). To ensure their efficacy, the PSF was placed in the spectrophotometer in front of the ray $(755 \mathrm{~nm})$ and the percentage of exiting light was determined. Then a PSF was placed vertically on the axis of the primary sheet to determine the rate of polarization generated in the presence of the PSF. The results demonstrated that using two parallel sheets was adequate for polarizing more than $99 \%$ of photons with a $755 \mathrm{~nm}$ wavelength. The rate of reduction in the light intensity due to the placement of PSF was measured and calculated by the spectrophotometer.

\section{Procedure}

Each patient was treated for a period of 2 weeks, 5 sessions a week. At the first session, the research objective was explained completely to the voluntary participants who 
met the inclusion criteria. Pain intensity was measured by a self-reported VAS score. The patients were asked to mark their pain level on a scale of $0-100$. Using this scale, pain intensity was measured at a resting position at the beginning of the 1st treatment session and at the end of the 5th and 10th sessions. Thirty-six participants had a trigger point in the right trapezius muscle and 22 had a trigger point in the left trapezius muscle. On average, each patient had 5 trigger points treated. In addition, the assessor evaluated the presence or absence of RP, the limitation of neck movement (LNM), the presence of muscular taut band (PMTB), and the sensitivity of muscular taut band (SMTB). The study was conducted in a double-blind method. The participants and the assessor were blind to the treatment modality. The blind setting for the data analyst was done by simply labeling the groups. The data analyst was unaware of the code for PLLLT and LLLT.

The patients were asked to avoid using analgesics, muscle relaxants, or local anesthetic medications. As the instances of noncompliance were reported or detected even during a single treatment session, the patient was automatically dismissed from the research. Each pain area and the taut band were treated with a laser beam of $6 \mathrm{~J} /$ $\mathrm{cm}^{2}$ intensity. This research employed the poly laser Trion LASER system (Sperian Co., Germany) in a continuous mode. The exit power of the system was $160 \mathrm{mw}$ at a 755 nm wavelength.

\section{Statistical Analysis}

The obtained figures of the VAS from the 1st, 5th and 10th sessions were evaluated via two-way repeated measures ANOVA and Tukey multiple comparisons test at a $5 \%$ significance level. In addition; the data from the assessor's examination (the presence of RP, LNMs, the presence and sensitivity of the taut band MTrPs) at the 1st, 5th and 10th sessions due to two treatment methods were investigated by the use of the Pearson chi-square test and the Fisher exact test. IBM SPSS Statistics 20 (SPSS Inc., Chicago, USA) was used for all statistical calculations.

\section{Results}

Table 1 presents the data regarding the rate of pain from the research participants compared to the specifications of the disease. All of the patients exhibited PR, LNM, PMTB and SMTB. Six patients were dismissed due to noncompliance criteria. Finally, the data from the 28 persons in the LLLT group and 30 people in the PLLLT group were reviewed and analyzed (Figure 1).

Table 2 displays the data collected by VAS about pain intensity. These data were analyzed by two-way repeated measures ANOVA. Evaluation between the sessions comparing PLLLT and LLLT outcomes was significant $(P<0.0001, d f=2, \mathrm{~F}=335.99)$. The results demonstrated that increasing the number of treatment sessions was effective in reducing pain intensity. Separate repeated
Table 2. Comparison of the Mean and Variance of Pain Intensity During 1st, 5th and 10th Sessions Due to Treatment Methods

\begin{tabular}{lccc}
\hline & 1st Session & 5th Session & 10th Session \\
\hline PLLLT & $78 \pm 9$ & $67 \pm 10$ & $55 \pm 16$ \\
LLLT & $78 \pm 10$ & $47 \pm 14$ & $12 \pm 14$ \\
\hline
\end{tabular}

PLLLT: polarized low-level therapy laser, LLLT: low-level therapy laser. Pain intensity was measured by visual analog scale (VAS).

measurement tests for the LLLT and PLLLT groups reported statistically significant effects. The reduced rate of pain intensity by the 2 techniques, LLLT and PLLLT, was also significant. The impact of LLLT on pain reduction was significantly greater than that of PLLLT $(P<0.0001$, $d f=1, F=64.84)$. The Tukey's multiple comparison test at the end of the 10th session demonstrated that LLLT was the more effective method (mean of pain intensity $=$ $12 \pm 14 \mathrm{~mm}$ ). The interaction of treatment methods and treatment sessions was also significant $(P<0.0001, d f=2$, $\mathrm{F}=78.79$ ).

Analysis of the results by Pearson's chi-square and Fisher exact tests at the completion of the 10th session showed that the LLLT and PLLLT groups in the presence or absence of RP, LNM, PMTB and SMTB had a significant difference (Table 3); the treatment of the subjects by LLLT was more effective than their treatment by PLLLT.

\section{Discussion}

The patients were divided into two groups in this research. As seen in Table 1, age distribution, gender, duration and intensity of the pain are almost equal. This table shows that the treatment groups were similar to each other before the intervention of this research.

Analysis of pain intensity by the use of VAS at the end of the 5th and 10th sessions in comparison to the 1st session showed that both treatment methods, LLLT and PLLLT, were effective in reducing pain intensity $(P<0.01$, $d f=2, \mathrm{~F}=335.99$ ); however, despite the observation of improvement in the PLLLT group, its effectiveness in reducing pain intensity was less compared to the LLLT

Table 3. Comparison of Patient Distribution According to the Presence of Signs/Symptoms Following the 10th Visit Due to Treatment Methods

\begin{tabular}{lcccc}
\hline & & $\begin{array}{c}\text { LLLT } \\
\text { No. }(\%)\end{array}$ & $\begin{array}{c}\text { PLLLT } \\
\text { No. }(\%)\end{array}$ & P Value \\
\hline Radicular pain & Yes & $0(0)$ & $16(53.3)$ & $0.0001^{*}$ \\
& No & $28(100)$ & $14(46.7)$ & \\
Limitation of neck & Yes & $1(3.6)$ & $12(40)$ & $0.001^{*}$ \\
movement & No & $27(96.4)$ & $18(60)$ & \\
Taut muscular band & Yes & $2(7.1)$ & $24(80)$ & $0.0001^{*}$ \\
Sensitivity of the taut & Yes & $26(92.9)$ & $6(20)$ & \\
muscular band & No & $26(7.1)$ & $22(73.3)$ & $0.0001^{*}$ \\
\hline
\end{tabular}

PLLLT: polarized low-level therapy laser, LLLT: low-level therapy laser, n: Number.

* The chi-square test and the Fisher exact test show a significant change $(P<0.05)$ between the two treatment methods. 
group. The difference between LLLT and PLLLT was completely significant $(P<0.001, d f=1, \mathrm{~F}=64.84)$. In other words, LLLT is preferable to PLLLT in reducing pain. The superior results of LLLT in reducing pain, in accordance with our VAS scores, agree with many reports that investigated the effect of laser light on reducing pain. ${ }^{23,34,35}$

Uemoto et $\mathrm{al}^{36}$ investigated the effect of laser therapy and needling on deactivating MTrPs. In this research, in 4 sessions of laser irradiation at wavelength $=795 \mathrm{~nm}$ at power $=80 \mathrm{~mW}$ and an exposure rate of $4 \mathrm{~J} / \mathrm{cm}^{2}$, a significant pain reduction compared to a control group was observed. In the present study, after 5 exposures of LLLT, a significant reduction in MTrPs was observed. However, increasing the number of sessions to 10 led to a greater pain reduction; in our research in the LLLT group, 12 patients had a VAS $=0$, and 16 patients, VAS was $<10$ after 10 treatment sessions. After observing RP at the 1st session in all 28 participants, at the end of the 10th session there was no RP in any of them, even though after the 5 th session, in spite of significant relief of RP in LLLT, 13 patients still complained of RP. Perhaps the significant reduction of pain with the increased numbers of sessions indicates that at least 10 therapeutic sessions should be routine in LLLT. ${ }^{11,27,37}$

The assessments of the effectiveness of LLLT and PLLLT in RP, LNM, PMTB and SMTB pain reduction were statistically significant. However, Table 3 demonstrates that the effectiveness of LLLT was higher than that of PLLLT. More than $90 \%$ of the patients in the LLLT group had none of the above-mentioned problems after the 10th session. In the PLLLT group, improved neck movement was observed in $60 \%$ of participants. Admittedly, this figure shows a lesser degree of effectiveness than the 90\% improvement via LLLT; the elimination of pain was achieved in less than $50 \%$ of PLLLT participants.

The acquired results of the LLLT irradiation were similar to the results of Shahimoridi et $\mathrm{al}^{37}$ who assessed the effect of LLLT at an $810 \mathrm{~nm}$ wavelength with $8 \mathrm{~J} /$ $\mathrm{cm}^{2}$ on trigger points of myofascial trapezius muscles. This research revealed that LLLT treatment in $70 \%$ of cases relieved the taut muscular band; $83 \%$ experienced a reduction of $\mathrm{RP}$ and $70 \%$ had an improvement in movement. The effectiveness of the $755 \mathrm{~nm}$ laser in our research was relatively greater than that of Shahimoridi et al. Perhaps the primary reason was our use of an $8 \mathrm{~J} / \mathrm{cm}^{2}$ irradiation dose. Investigators who used $4-6 \mathrm{~J} / \mathrm{cm}^{2}$ doses had good effectiveness. For example, Uemoto et al, ${ }^{36}$ who chose irradiation of $795 \mathrm{~nm}$ and a $4 \mathrm{~J} / \mathrm{cm}^{2}$ dose, reported more effect compared to irradiation with $8 \mathrm{~J} / \mathrm{cm}^{2}$ on the reduction of MTrPs.

Still, why was the polarized laser less effective than the non-polarized laser? In our experience, if a diverse mechanism cannot be identified for greater diminishment of pain by the polarized laser, one would observe at least the same effect as LLLT because mechanisms that could account for the effect of LLLT on pain reduction include the improvement of small area blood circulation, leading to an increased oxygen source for hypoxic cells at trigger point areas. ${ }^{26}$ The reason for observing such an effect may be in the irradiation time of each session. The power of the laser system was constant and exposed an equal irradiation dose to trigger points while the PSF was in front of the laser. This approach deleted half of the irradiated light in transmittance through the PSF. The exposure time would have to be doubled to generate a dose to the trigger points equal to that of LLLT.

Perhaps the reason for the higher impact in the LLLT group is that the desirable dose was reached in less time because reports also exist about no pain improvement from laser irradiation. Dundar et $\mathrm{al}^{38}$ assessed the effect of a gallium-aluminum-arsenide (Ga-Al-As) laser at an $830 \mathrm{~nm}$ wavelength on trigger points during 15 treatment sessions, in which no significant effect on pain reduction was observed in comparison with the control group. In their study, both the control group and the laser treatment group had daily isometric and stretching exercises. The exit power of their laser system was 58 $\mathrm{mW} / \mathrm{cm}^{2}$ when the irradiated dose on each point was 7 J. Altan et $\mathrm{al}^{13}$ also reported a similar lack of effectiveness of LLLT in reducing MP. In their research, a Ga-As laser at a $904 \mathrm{~nm}$ wavelength was used. All patients in both laser irradiation groups as well as the control group had been trained in daily isometric exercises and experienced only short pain-free periods for 2 weeks at home. Thus, both groups experienced some improvement, but they had no discernable difference when compared with each other. Thorsen et $\mathrm{al}^{39}$ assessed the effect of Ga-Al-As laser light at an $830 \mathrm{~nm}$ wavelength and $30 \mathrm{~mW}$ power on neck and back myofascial muscles with an irradiation dose of $9 \mathrm{~J}$, but no difference between the placebo and irradiated groups was observed. Comparing the specified dose of the laser used with the above-mentioned groups and those groups with positive effects from LLLT ${ }^{11}$ points to differences in the use of extremely low exit power by those researchers who sustained the above hypothesis.

In some studies, no significant difference was observed upon comparing the effectiveness of LLLT and other treatment techniques. ${ }^{11,40,41}$ However, the findings of this research evaluating the effect of LLLT in comparison with PLLLT on pain reduction of MTrPs were completely significant, consistent with the findings from a study by Demirkol et al. ${ }^{11}$ As it is shown in Table 3, the results of the present study regarding the presence or absence of RP, LNM, PMTB and SMTB upon the completion of 10 sessions showed a significant difference between the two treatment methods in such a way that LLLT had a greater impact than PLLLT.

\section{Conclusion}

PLLLT and LLLT are effective in treating patients presenting with MTrPs in the trapezius muscles. However, 
the effectiveness of LLLT was significantly superior to PLLLT. 10 sessions of LLLT caused improvement in RP, LNM, PMTB and SMTB in more than $90 \%$ of the patients, significantly more effective than PLLLT. Given the observed results, we recommend LLLT as a preferred method for the treatment of MTrPs.

\section{Ethical Considerations}

The research protocol was approved by the Ethics Committee of Rafsanjan University of Medical Sciences and was registered in the Iranian Registry of Clinical Trials (identifier: IRCT2015081623647N1; https://www. irct.ir/trial/20154).

\section{Conflict of Interests}

The authors declare no conflict of interest.

\section{Acknowledgment}

This research was supported by grants from Rafsanjan University of Medical Sciences. We give special thanks to Z. Assadollahi for helping in data analysis and S. Skies for editing this manuscript.

\section{References}

1. Fricton JR. Clinical care for myofascial pain. Dent Clin North Am. 1991;35(1):1-28.

2. Cummings M, Baldry P. Regional myofascial pain: diagnosis and management. Best Pract Res Clin Rheumatol. 2007;21(2):367-87. doi: 10.1016/j.berh.2006.12.006

3. Cheshire WP, Abashian SW, Mann JD. Botulinum toxin in the treatment of myofascial pain syndrome. Pain. 1994;59(1):65-69. doi: 10.1016/0304-3959(94)90048-5

4. Celiker R, Atalay A, Guven Z. Health-related quality of life in patients with myofascial pain syndrome. Curr Pain Headache Rep. 2010;14(5):361-6. doi: 10.1007/s11916-0100141-7

5. Dommerholt J, Huijbregts P. Myofascial trigger points: pathophysiology and evidence-informed diagnosis and management. Sudbury, Massachusetts: Jones and Bartlett Publishers; 2011.

6. Desai MJ, Saini V, Saini S. Myofascial pain syndrome: a treatment review. Pain Ther. 2013;2(1):21-36. doi: 10.1007/ s40122-013-0006-y

7. Charles D, Hudgins T, MacNaughton J, Newman E, Tan J, Wigger M. A systematic review of manual therapy techniques, dry cupping and dry needling in the reduction of myofascial pain and myofascial trigger points. $J$ Bodyw Mov Ther. 2019;23(3):539-546. doi:10.1016/j. jbmt.2019.04.001

8. Zhang XF, Liu L, Wang BB, Liu X, Li P. Evidence for kinesio taping in management of myofascial pain syndrome: a systematic review and meta-analysis. Clin Rehabil. 2019;33(5):865-874. doi:10.1177/0269215519826267

9. Khalighi HR, Mortazavi H, Mojahedi SM, Azari-Marhabi S, Moradi Abbasabadi F. Low level laser therapy versus pharmacotherapy in improving myofascial pain disorder syndrome. J Lasers Med Sci. 2016;7(1):45-50. doi:10.15171/ jlms.2016.10

10. Momenzadeh S, Akhyani V, Razaghi Z, Ebadifar A,
Abbasi M. Evaluation of the effects of intravenous and percutaneous low level laser therapy in the management of shoulder myofascial pain syndrome. J Lasers Med Sci. 2016;7(1):16-20. doi: 10.15171/jlms.2016.04

11. Demirkol N, Sari F, Bulbul M, Demirkol M, Simsek I, Usumez A. Effectiveness of occlusal splints and lowlevel laser therapy on myofascial pain. Lasers Med Sci. 2014;30(3):1007-12. doi: 10.1007/s10103-014-1522-7.

12. Kannan P. Management of myofascial pain of upper trapezius: a three group comparison study. Glob J Health Sci. 2012;4(5):46-52. doi: 10.5539/gjhs.v4n5p46

13. Altan L, Bingöl U, Aykaç M, Yurtkuran M. Investigation of the effect of GaAs laser therapy on cervical myofascial pain syndrome. Rheumatol Int. 2005;25(1):23-27. doi: 10.1007/ s00296-003-0396-y

14. Burger M, Kriel R, Damon A, et al. The effectiveness of lowlevel laser therapy on pain, self-reported hand function, and grip strength compared to placebo or "sham" treatment for adults with carpal tunnel syndrome: A systematic review. Physiother Theory Pract. 2017;33(3):184-197. doi: 10.1080/09593985.2017.1282999

15. Togsverd-Bo K. Early laser intervention for improved appearance of surgical scars. Br J Dermatol. 2019;180(1):25. doi: 10.1111/bjd.17358

16. Pereira TRC, Vassão PG, Venancio MG, Renno ACM, Aveiro MC. Non-ablative radiofrequency associated or not with low-level laser therapy on the treatment of facial wrinkles in adult women: a randomized single-blind clinical trial. J Cosmet Laser Ther. 2017;19(3):133-9. doi:1 0.1080/14764172.2016.1269929

17. Yildirimturk S, Sirin Y, Soluk Tekkesin M, Gurler G, Firat D. The effects of low-level laser therapy on the healing of bone defects in streptozotocin-induced diabetic rats: a histological and morphometric evaluation. J Cosmet Laser Ther. 2017;19(7):397-403. doi: 10.1080/14764172.2017.1341048

18. Naghdi S, Ansari NN, Fathali M, Bartley J, Varedi M, Honarpishe R. A pilot study into the effect of low-level laser therapy in patients with chronic rhinosinusitis. Physiother Theory Pract. 2013;29(8):596-603. doi: 10.3109/09593985.2013.775204

19. Dastanpour S, Momen Beitollahi J, Saber K. The effect of low-level laser therapy on human leukemic cells. J Lasers Med Sci. 2015;6(2):74-79.

20. Yousef M, Mansouri P, Partovikia M, Esmaili M, Younespour S, Hassani L. The effect of low level laser therapy on Pemphigus vulgaris lesions: a pilot study. J Lasers Med Sci. 2017;8(4):177-180. doi:10.15171/jlms.2017.32

21. Imani F, gharehdaghi FA. Review articles: Efficacy of Low Level Laser Therapy in fibromyalgia and myofacial pain syndrome. Journal of Anesthesiology and Pain. 2011;2(5):111. [Persian]

22. Munguia FM, Jang J, Salem M, Clark GT, Enciso R. Efficacy of low-level laser therapy in the treatment of temporomandibular myofascial pain: A systematic review and meta-analysis. J Oral Facial Pain Headache. 2018;32(3):287-97. doi: 10.11607/ofph.2032.

23. Momenzadeh S, Akhyani V, Razaghi Z. Evaluation of the effects of intravenous and percutaneous low level laser therapy in the management of shoulder myofascial pain syndrome: A controlled randomized clinical trial. J Lasers 
Med Sci. 2016;7(1):16-20. doi: 10.15171/jlms.2016.04.

24. Snyder-Mackler L, Barry AJ, Perkins AI, Soucek MD. Effects of helium-neon laser irradiation on skin resistance and pain in patients with trigger points in the neck or back. Phys Ther. 1989;69(5):336-41. doi: 10.1093/ptj/69.5.336

25. Mirbod SM, Ghahremani P, Malekian M, Sarrami H, Ebnshahidi N. The effect of low-level laser therapy on acupuncture points in patients with myofascial pain syndrome. Journal of Research in Rehabilitation Sciences. 2010;6(2):1-10. [Persian].

26. Simunovic Z. Low level laser therapy with trigger points technique: A clinical study on 243 patients. J Clin Laser Med Surg. 1996;14(4):163-7. doi: 10.1089/clm.1996.14.163

27. Bahrami Mh, Mokhtari MR, Rayegani M, et al. Effect of laser and ultrasound treatments on myofascial pain syndrome: a comparative study. Shahid Beheshti University of Medical Sciences. 2009;69(3):129-135. [Persian].

28. ADA council on scientific affairs. Direct and indirect restorative materials. J Am Dent Assoc. 2003;134(4):463472. doi: 10.14219/jada.archive.2003.0196

29. Huang D, Gu YH, Liao Q, Yan XB, Zhu SH, Gao CQ. Effects of linear-polarized near-infrared light irradiation on chronic pain. Scientific WorldJournal. 2012;2012:567496. doi: 10.1100/2012/567496.

30. Limansky YP, Tamarova ZA, Gulyar SA. Suppression of pain by exposure of acupuncture points to polarized light. Pain Res Manag. 2006;11(1):49-57. doi: 10.1155/2006/784968

31. Liao CD, Rau CL, Liou TH, Tsauo JY, Lin LF. Effects of linearly polarized near-infrared irradiation near the stellate ganglion region on pain and heart rate variability in patients with neuropathic pain. Pain Med. 2017;18(3):488503. doi:10.1093/pm/pnw145

32. Durović A, Marić D, Brdareski Z, Jevtić M, Durdević S. The effects of polarized light therapy in pressure ulcer healing. Vojnosanit Pregl. 2008;65(12):906-912. doi:10.2298/ vsp0812906d

33. Monstrey SJ, Hoeksema H, Saelens H, et al. A conservative approach for deep dermal burn wounds using polarisedlight therapy. Br J Plast Surg. 2002;55(5):420-426. doi:http:// dx.doi.org/10.1054/bjps.2002.3860

34. Agung I, Murdana N, Purba H, Fuady A. Low-level laser therapy and dry needling for myofascial pain syndrome of the upper trapezius muscle: An interventional study. J Phys Conf. 2018; 1073(6):062045 doi: 10.1088/17426596/1073/6/062045

35. Altindiş T, Güngörmüş M. Thermographic evaluation of occlusal splint and low level laser therapy in myofascial pain syndrome. Complement Ther Med. 2019;44:277-81. doi: 10.1016/j.ctim.2019.05.006

36. Uemoto L, Garcia MA, Gouvea CV, Vilella OV, Alfaya TA. Laser therapy and needling in myofascial trigger point deactivation. J Oral Sci. 2013;55(2):175-81. doi:10.2334/ josnusd.55.175

37. Shahimoridi D, Eghbali M, Vaziri Nejad R, Najafzadeh N. Using Low Power Laser in the Treatment Shahimoridi of the Myofascial Trigger Points. Journal of Rafsanjan University Of Medical Sciences. 2009;9(2):99-108. [Persian]

38. Dundar U, Evcik D, Samli F, Pusak H, Kavuncu V. The effect of gallium arsenide aluminum laser therapy in the management of cervical myofascial pain syndrome: a double blind, placebo-controlled study. Clin Rheumatol. 2007;26(6):930-934. doi:10.1007/s10067-006-0438-4

39. Thorsen H, Gam AN, Svensson BH, et al. Low level laser therapy for myofascial pain in the neck and shoulder girdle. A double-blind, cross-over study. Scand J Rheumatol. 1992;21(3):139-41. doi: 10.3109/03009749209095086

40. Oz S, Gokcen-Rohlig B, Saruhanoglu A, Tuncer EB. Management of myofascial pain: low-level laser therapy versus occlusal splints. J Craniofac Surg. 2010;21(6):17221728. doi: 10.1097/SCS.0b013e3181f3c76c

41. Kiralp MZ, Ari H, Karabekir I, Dursun H. Comparison of low intensity laser therapy and trigger point injection in the management of myofascial pain syndrome. The Pain Clinic. 2006;18(1):63-6. doi: 10.1163/156856906775249794 